\title{
Enhanced electrochemical performance of ion-beam-treated 3D graphene aerogels for lithium ion battery
}

\author{
J.C. Ye, S. Charnvanichborikarn, M.A. Worsley, S.O. Kucheyev, B.C. Wood, Y.M. Wang* \\ Physical and Life Sciences Directorate, Lawrence Livermore National Laboratory, Livermore, \\ CA 94550, USA
}

\begin{abstract}
High energy light-ion (3.8 MeV He) bombardment is used to introduce lattice defects in a 3dimensional (3D) interconnected network of graphene aerogels (GAs). When these materials are used as anodes for lithium ion battery, we observe improved percentage reversible capacity and cycle stability compared to those without ion-beam treatment. Furthermore, all ion-beam treated 3D graphene samples exhibit substantially higher Coulombic efficiencies, suggesting at beneficial role of vacancy-type defects in stabilizing solid-electrolyte interphases. Although 3D graphene exhibits initial reversible capacities that are 2-3 times higher than that of graphite $(\sim 372 \mathrm{mAh} / \mathrm{g})$, fast capacity fading is observed but becomes more stable after ion-beam treatment. Our experimental results demonstrate that ion-beam treatment is an effective route to tune and produce good-performance graphene electrodes, and that vacancy-type defects help to promote reversible lithium storage capacity in graphene. We further observe that 3D GAs irradiated to the highest dose studied $\left(10^{16} \mathrm{~cm}^{-2}\right)$ fail rapidly upon electrochemical cycling, likely caused by the excessive ion-beam damage and graphene restacking. Raman I(D)/I(D') signature is considered linked to defect type in graphene and thus is proposed, for the first time, as an indicator of the reversible capacity for GAs.
\end{abstract}

Keywords: graphene; lithium-ion battery; ion-beam treatment; graphene defects; Raman spectroscopy

*Corresponding author email: ymwang@1lnl.gov 


\section{Introduction}

Although graphene has been broadly investigated, both theoretically and experimentally, for applications in lithium ion batteries (LIBs) (as well as supercapacitors) [1-4], the fundamental lithium-storage mechanisms in graphene and their reversibility remain largely unresolved. In addition to well-recognized interlayer lithium storage sites [5], a variety of structural defects in graphene are considered important for lithium storage [3]. First-principles density functional theory calculations $[6,7]$ have for example revealed that the presence of vacancies, dislocations, and ad-dimers in graphene can activate otherwise unfavorable surface binding sites for lithium (Table 1). On the other hand, binding on Stone-Wales and heptagon defects is unstable with respect to lithium cluster formation, meaning the storage capacity of defective graphene depends on the specific defect identity. Some defects — notably edges and unpassivated monovacancies that involve $\sigma$-type bonding of lithium - bind too strongly, such that the associated capacity increase is unlikely to be reversible. Experimentally, however, definitive correlation between the structural defects of graphene and their reversible lithium storage capacity has not been well understood. Challenges associated with experiments include the formation of solid-electrolyte

interphases (SEIs), which is inevitable for LIB applications due to the work function mismatch of carbon/graphene and the lowest unoccupied molecular orbital (LUMO) of commercial electrolytes $\left(\mathrm{LiPF}_{6}\right)$ [8], and the facts that graphene has very large surface area (with a theoretical value of $2630 \mathrm{~m}^{2} / \mathrm{g}$ ), leading to restacking and folding due to strong $\pi$ - $\pi$ interactions during continuous charging/discharging cycles and a loss of reversible capacity. Due to the SEI formation, it has been suggested by some experiments that the surface defects of graphene should be considered as nonreversible storage sites despite their favorable binding energy with lithium [3], whereas internal defects are considered as reversible storage sites. Other experiments $[9,10]$, which often use polymeric binders for electrode fabrication, argue instead that surface nanocavities or even surface adsorption on both sides of graphene could contribute to the reversible lithium capacity. Nevertheless, these oversimplified experimental arguments based on the geometric location of defects are in stark contrast to mechanistic simulations in which models are mainly constructed to explore the nature of defects. This obvious gap between theories and experiments (as well as ongoing experimental controversies) calls for a critical need for wellcontrolled studies in order to elucidate the realistic roles of defects in promoting (or degrading) lithium storage capability of graphene. 
Vacancies are perhaps the simplest point defects in graphene. Aside from critically influencing the electronic, mechanical, and catalytic properties [11, 12], vacancies are also predicted by theory to play an important role for lithium storage $[6,13]$. Due to the juxtaposed effects of complex defect structures (e.g., edges, $\mathrm{sp}^{3}$-type defects, grain boundaries) in many graphene derivatives, however, it has been long challenging to isolate the specific roles of vacancies in lithium storage capability. As a result, computer simulations have been hitherto the primary avenue for studying the vacancy-related storage mechanisms [6, 13]. To the best of our knowledge, there are no existing experimental studies that can directly link vacancies to the energy storage ability of graphene despite the favorable theoretical predictions (see references [6, 7, 13] and Table 1).

Recent in-situ TEM studies [14] have highlighted additional potential engineering advantages of graphene, which was found mechanically more robust than carbon nanotubes after lithiation/delithiation, making it an ideal building block for 3-dimensional (3D) porous network. Some experimental work has indeed emerged to take advantage of this excellent property of graphene $[4,15,16]$. However, most these studies have been focused on supercapacitor-type applications [1, 15], where surface phenomena dominate. Key questions remain as to whether 3D porous graphene network can sustain much harsher LIB conditions where lithium diffusion related bulk behavior becomes critical, and how one can enhance the LIB performance for 3D graphene network.

In this study, $3 \mathrm{MeV} \mathrm{He}$ ion bombardment is applied to introduce lattice defects in highquality, 3D network of graphene aerogels (GAs). We choose He-ions here instead of heavier ions (e.g., $\mathrm{Ne}^{+}, \mathrm{Ar}^{+}$or $\mathrm{Xe}^{+}$) as our previous work on carbon nanotube aerogels $[17,18]$ suggests that heavy ions cause serious densification of carbon aerogels, change of surface areas, and thus complicate electrochemical performance studies. Our as-synthesized GAs comprise strongly cross-linked 3D graphene network, which has good electrical conductivity and structural integrity against electrochemical charge/discharge, thus eliminating the need for additional binders/additives. This helps to facilitate mechanistic studies. When ion beam treated 3D GAs are used as anodes for LIBs, we observe some surprising improvement in their performance. Specially, vacancy-type defects are found to simultaneously help improve the percentage reversible capacity, Coulombic efficiency of 3D graphene and long-term cyclability. 
Additionally, we observe that 3D GAs are relatively resistant to $\mathrm{He}^{+}$beam damage up to a certain dose level after which the 3D structure starts to deteriorate under electrochemical environment.

\section{Experimental Section}

\subsection{Preparation of 3D GAs and surface area measurements}

In order to avoid the complications caused by polymeric binders and carbon black that are commonly used to fabricate graphene-based electrodes $[5,10]$, it is important that the starting graphene materials are carbon black free and binder free. To this end, we fabricate thick 3D nanoporous GAs by gelation of graphene oxide (GO) slurry in base solution followed by supercritical drying and pyrolysis at $1050{ }^{\circ} \mathrm{C}$. The details of fabrication processes have been reported in our previous work $[19,20]$. In short, single layered GO nanoribbons with a length of $\sim 500 \mathrm{~nm}$ were purchased from Cheap Tubes Inc. (Brattleboro, VT 05301, USA). $300 \mathrm{mg}$ of GO was dissolved in $15 \mathrm{ml}$ deionized water with ultrasonication overnight, after which $3.165 \mathrm{ml}$ of concentrated $\mathrm{NH}_{4} \mathrm{OH}$ was added in the $\mathrm{GO} / \mathrm{H}_{2} \mathrm{O}$ slurry. A proper amount of the above slurry was cast in a 12.5-mm-in-diameter circle-shaped mold capped by two glass slides and spaced by 0.5mm-thick rubber sheet. It was submerged in a beaker full of deionized water and placed in an oven at $85^{\circ} \mathrm{C}$ for $72 \mathrm{hrs}$. The obtained wet gel was washed in deionized water to remove reaction by-products, and exchanged with acetone. Acetone in the wet gel pores was exchanged for liquid $\mathrm{CO}_{2}$, after which the temperature of the vessel was ramped up to $50{ }^{\circ} \mathrm{C}$, with the vessel pressure maintained above $1.2 \mathrm{ksi}$. The vessel was returned to atmospheric pressure to remove the dried monolith, which was further annealed at $1050{ }^{\circ} \mathrm{C}$ under $\mathrm{N}_{2}$ flow for $3 \mathrm{hrs}$. To obtain better crystalline graphene [21], the initial GAs were further annealed in an alumina furnace at $1600{ }^{\circ} \mathrm{C}$ under argon (99.99+\% purity) flow for 4 hours. The resultant GAs (denoted as 1600C-GAs) are $250 \mu \mathrm{m}$ thick, $\mathrm{sp}^{2}$-bonded 3D porous structure with high electrical conductivity (better than $\sim 2.5$ S/cm), good chemical stability and mechanical robustness, making them suitable for LIB applications. The specific surface area was determined by nitrogen adsorption/desorption using the Brunauer-Emmett-Teller (BET) method, with an ASAP 2020 surface area analyzer (Micromeritics Instrument Corp.). The BET isotherm curves and pore size distribution of our GA-1600C are shown in Supplementary Data.

\section{2 $\mathrm{He}^{+}$ion bombardment}


The 1600C-GA samples were mounted on silicon substrates and bombarded at room temperature using $3.8 \mathrm{MeV} \mathrm{He}^{+}$with a constant beam flux of $10^{12} \mathrm{~cm}^{-2} \mathrm{~s}^{-1}$ at an incident angle normal to the sample surface to three different doses of $10^{14}, 10^{15}$, and $10^{16} \mathrm{~cm}^{-2}$, respectively (hereinafter referred to as He-14, He-15, and He-16 samples, respectively). Depth distributions of atomic displacements and implanted He atoms were calculated with the TRIM code (version SRIM2011.08) [22], with the threshold displacement and lattice binding energies of $28 \mathrm{eV}$ and $3 \mathrm{eV}$, respectively. Note that the thickness of the $70 \mathrm{mg} / \mathrm{cm}^{3}$ carbon sample discs used in this study is $250 \mu \mathrm{m}$, which is much smaller than the projected ion range of $\sim 400 \mu \mathrm{m}$. Results of TRIM code simulations shown in Fig. 1 indicate that GA samples have received relatively uniform displacements throughout the depth (i.e., $0-250 \mu \mathrm{m}$ in the figure) while the $\mathrm{He}$ ions were transmitted through GAs and implanted into the silicon or sapphire substrate.

To measure the radiation-induced change of electrical resistance, the sample discs were placed on sapphire instead of silicon substrate and the conductive silver paste was used to make electrical contacts. The conductivity was measured after each dose increment, with the sample remaining under vacuum.

\subsection{Raman spectroscopy, scanning electron microscopy (SEM), and transmission electron microscopy (TEM)}

The structures of 3D graphene were investigated by Micro-Raman spectroscopy (Nicolet Almega XR Dispersive) with an excitation wavelength of $633 \mathrm{~nm}(1.96 \mathrm{eV})$, a spot size of $800 \mathrm{~nm}$ (100x), and a resolution of $\sim 2 \mathrm{~cm}^{-1}$. An ultralow laser beam power of $<0.1 \mathrm{~mW}$ was applied to avoid laser heating or damage on GAs, as a high power laser beam could trigger desorption of adatoms from graphene. To avoid the structural nonuniformity of GAs, at least three Raman spectra were collected from different areas of each sample. The Raman peaks are fitted with Lorentzian functions. We denote the peak intensity (i.e., height) as $I(D), I(G)$, and $I\left(D^{\prime}\right)$. Different ratios of peak intensity are subsequently used to interpret the disorder structures introduced by $\mathrm{He}^{+}$beam treatment.

The GAs morphology was investigated by SEM (JOEL 7401) operated at an accelerating voltage of $8 \mathrm{kV}$. A Philips CM30 field-emission TEM was applied to characterize the nanometer scale features of 3D graphene, with a point-to-point spatial resolution of $1.8 \AA$. The TEM samples were prepared by manually crashing the 3D GAs onto lacey-carbon copper grids (200 
mesh, Ted Pella). Standard bright-field and high-resolution TEM images were acquired for morphology analysis.

\subsection{Electrochemical characterizations}

$250 \mu \mathrm{m}$ thick GA discs with a diameter of $\sim 4 \mathrm{~mm}$ [inset Fig. 2(a)] were directly used as the working electrodes in Swagelok-type half-cells. For graphite electrodes, a mixture of graphite powders (MTI Corp., particle size $\sim 0.5-5 \mu \mathrm{m}$ ) and carbon black (Super C65) was dispersed in 1.25 wt.\% polyvinylidene difluoride (PVDF)/N-methyl-2-pyrrolidone (NMP) solution with a weight ratio of 7 (graphite): 2(Super C65): 1(PVDF). It was then spread on copper foil using a knife blade. The electrode was dried at $80{ }^{\circ} \mathrm{C}$ under vacuum overnight. Discs with $6 \mathrm{~mm}$ in diameter were punched out for electrochemical measurements. Lithium chips with a diameter of $\sim 9 \mathrm{~mm}$ were used as counter electrodes and $1 \mathrm{M} \mathrm{LiPF}_{6}$ in ethyl carbonate (EC)/diethyl carbonate (DEC)/dimethyl carbonate (DMC) (1:1:1 in volume ratio) purchased from MTI Cor. as the electrolyte. Since the formation of SEI layers is intimately linked to the type of electrolyte, we show in Table 2 the physical properties of the commercial electrolyte [23] used in our experiments. A double layer porous polypropylene film (Celgard 3501) was applied as the separator. The cells were assembled in Ar-filled glove box with very low contamination of oxygen and water content $(<1 \mathrm{ppm})$. Galvanostatic charge/discharge cycles were performed at a current density of $50 \mathrm{~mA} / \mathrm{g}$ in a voltage window of 0.01-3.5 V.

\section{Results and discussion}

\subsection{Structure of 3D GAs before and after $\mathrm{He}^{+}$ion beam treatment}

The top-view surface morphologies of 3D GAs before and after $\mathrm{He}^{+}$treatment at different doses are shown in Fig. 2(a)-(d). The sheet-like morphology, stacking nature, and porous structures of $3 \mathrm{D}$ graphene are visible in all these samples. The ion-beam treatment at low dose levels $\left(<2 \times 10^{15}\right.$ $\mathrm{cm}^{-2}$ ) does not appear to cause visible morphological damage of 3D graphene except for the slightly flattening of surface, Fig. 2(a), Fig. 2(b) and Fig. 2(c). When the dose level reaches $\sim 10^{16}$ $\mathrm{cm}^{-2}$, however, some voids-like features are observed, suggesting that high-dose $\mathrm{He}^{+}$flux could potentially degrade GAs. At the nanometer scales [insets of Fig. 2(b)-2(d)], we find that nanopores remain in 3D GAs. The BET measurements indicate that the as-synthesized 3D 1600C-GA has a total surface area of $\sim 1200 \mathrm{~m}^{2} / \mathrm{g}$ [21] (i.e., on average the GAs consist of $\sim 2.2$ layers of graphene sheets interconnected into 3D network). The surface area was found to reduce 
slightly after high-dose ion beam treatment $\left(\sim 10^{16} \mathrm{~cm}^{-2}\right)$ to a value of $937 \mathrm{~m}^{2} / \mathrm{g}$. This loss of accessible surface area may be related to the densification of 3D GAs. Note that the ion-beam treated samples are typically very small ( 1-3 mg), which may give a relatively large error bar in terms of BET surface measurement. For this primary reason, the surface area of He-14 and He15 were not measured.

For 1600C-GA, the TEM micrograph in Fig. 3(a) indicates that it comprises interconnected graphene nanosheets into 3D network. Wrinkling and curling features are visible, as well as sub$100 \mathrm{~nm}$ pores. High-resolution TEM in Fig. 3(b) reveals that the surface of graphene is mostly wrinkled but the flat region is visible, suggestive of graphitization at high annealing temperatures [21]. Stacked layers (typically 2-3 layers) are also observed with a measurable interlayer spacing (d) of $\sim 0.39 \mathrm{~nm}$, inset of Fig. 3(b) (i.e., larger than interlayer spacing of graphite $\sim 0.34 \mathrm{~nm}$ ). The enhanced $d$-spacing is commonly reported in graphene oxides reduced graphene materials and can be ascribed to structural defects or surface functional groups [5, 24]. We note in TEM that the stacking of graphene is not the dominant feature of 3D GAs, consistent with the BET measurements. The TEM and BET characterizations suggest that the structural defects of our 3D GAs are primarily located on the surface.

In order to understand the defect evolution and macroscopic degrading behavior of these materials under ion beam radiation and thus determine their suitability for LIBs, we monitor insitu the electrical conductivity of 3D GAs under different doses, Fig. 3(c). The electrical conductivity of 3D GAs remains fairly constant at $\sim 2.5 \mathrm{~S} / \mathrm{cm}$ up to $10^{14} \mathrm{He}^{+} / \mathrm{cm}^{2}$ dose level, where it decreases appreciably as the dose increases to $10^{15} \mathrm{He}^{+} / \mathrm{cm}^{2}$, but then increases with higher dose of $10^{16} \mathrm{He}^{+} / \mathrm{cm}^{2}$. The initial decrease of electrical conductivity is likely caused by defect accumulations in 3D GAs. Further ion dose to higher levels $\left(10^{16} \mathrm{~cm}^{-2}\right)$, however, leads to the densification of GAs and an increase of electrical conductivity. This behavior is different from what we have observed in carbon nanotube reinforced carbon aerogels [17], which shows continuous decrease of electrical conductivity in the same dose range of He ion.

We use Micro-Raman spectroscopy to characterize the defect evolution nature of 3D GAs under $\mathrm{He}^{+}$ion beam radiation. The Raman spectra of four types of samples are shown in Fig. 4(a), all of which exhibit disorder-induced D-band at $\sim 1350 \mathrm{~cm}^{-1}$, first-order Raman-allowed Gband at $\sim 1582 \mathrm{~cm}^{-1}$ (i.e., C-C stretching band), symmetry-allowed 2D band, defect-related D'- 
band, and the combination of D and D' bands (i.e., D+D' band). The G-band in all four samples shows a blue-shift to higher frequency values, suggestive of the strained state of graphene in 3D network. This agrees with the wrinkling and stacking features observed in TEM [Fig. 3(b)]. Other sources of strain such as curling of nanosheets in 3D network could also modify the bond lengths and angles of graphene and thus contribute to the G-band shift. We note that the starting 1600C-GA material has an $\mathrm{I}(\mathrm{D}) / \mathrm{I}(\mathrm{G})$ ratio of $2.67 \pm 0.18$, which continues to increase with higher $\mathrm{He}^{+}$doses, Fig. 4(a). This is mostly due to the increasing intensity of the disordered band [I(D)] caused by ion beam radiation. Although the defect evolution behavior in 3D graphene network under ion beam damage has never been studied before, some earlier Raman spectroscopy studies on well-controlled graphene sheets offer important clues on the nature of defect evolution in graphene under ion-beam $[25,26]$ or plasma exposure [11]. It has been generally agreed upon that the evolution of defects during graphene damage follows a two-stage process, separated by a critical graphene domain size $\mathrm{L}_{\mathrm{D}}\left(\mathrm{L}_{\mathrm{D}} \sim 3-4 \mathrm{~nm}\right)$; i.e., low defect density region (Stage 1, $\mathrm{L} \mathrm{L}_{\mathrm{D}}$ ) and high defect density region (Stage $2, \mathrm{~L}<\mathrm{L}_{\mathrm{D}}$ ). The exact value of this transition domain size $\left(\mathrm{L}_{\mathrm{D}}\right)$ currently remains under debate $[11,26]$.

Following the theory described in reference [25], the defect evolution under ion beam radiation can be generally described by the following equation,

$$
\frac{I(D)}{I(G)}=C_{A} \frac{\left(r_{A}^{2}-r_{S}^{2}\right)}{\left(r_{A}^{2}-2 r_{S}^{2}\right)}\left[e^{-\frac{\pi r_{S}^{2}}{L_{D}^{2}}}-e^{-\frac{\pi\left(r_{A}^{2}-r_{S}^{2}\right)}{L_{D}^{2}}}\right]
$$

Here $C_{\mathrm{A}}$ is the weighing parameter of an activated area $(\mathrm{A})$ and is related to the ratio between the scattering efficiency of optical phonons between $\mathrm{K}$ and $\Gamma . C_{A}=A E_{L}^{-B}$ decreases as the laser energy increases and was fit empirically from ion beam irradiation experiments with $A=(160 \pm$ 48) $\mathrm{eV}, \mathrm{B}=4$, and $\mathrm{E}_{\mathrm{L}}=1.96 \mathrm{eV}$ (i.e., laser energy) for our experiments. The other two important parameters $r_{A}$ and $r_{s}$ are length scales that determine the region where the D-band scattering occurs. Again, these two parameters were fitted from experiments as $r_{A}=3.1 \mathrm{~nm}$, and $r_{s}=1 \mathrm{~nm}$. Fig. 4(b) reproduces the $\mathrm{I}(\mathrm{D}) / \mathrm{I}(\mathrm{G})$ versus $\mathrm{L}_{\mathrm{D}}$ fitting plot, together with the $\mathrm{D}-\mathrm{G}$ band ratios measured from all of our samples. Note that our previous high temperature annealing results from $1500-2500{ }^{\circ} \mathrm{C}$ indicate that our GAs are Stage 1 materials with relatively large domain sizes, which increase with annealing temperature. Equation (1) can thus be simplified as 


$$
L_{D}^{2}\left(n m^{2}\right)=\frac{(4.3 \pm 1.3) \times 10^{3}}{E_{L}^{4}}\left(\frac{I(D)}{I(G)}\right)^{-1}
$$

We estimate a domain size of $10.4 \mathrm{~nm}$ for the as-synthesized 1600C-GA, which marginally drops down to $9.1 \mathrm{~nm}$ after the high dose of ion beam radiation $\left(\sim 10^{16} \mathrm{He}^{+} / \mathrm{cm}^{2}\right)$.

To further quantify the defect evolution behavior during our ion beam treatment, we apply the intensity ratio of the $\mathrm{D}$ and $\mathrm{D}^{\prime}$ peaks, which has been shown to be sensitive to the defect types in graphene [11]. Fig. 4(c) shows $I(D) / I(G)$ versus $I\left(D^{\prime}\right) / I(G)$ plot with the slopes [i.e., $\left.\mathrm{k}=\mathrm{I}(\mathrm{D}) / \mathrm{I}\left(\mathrm{D}^{\prime}\right)\right]$ of each type of defects delineated in the figure. Our initial material 1600C-GA has a $\mathrm{k}$-value of 5.49 , suggesting the co-existence of $\mathrm{sp}^{3}$-defects, vacancies and boundary-type defects. The $\mathrm{sp}^{3}$-defects could be caused by the surface functional groups adsorbed on graphene sheets or cross-linking $\mathrm{sp}^{3}$ carbon atoms in 3D network. As many functional groups are preferentially adsorbed at the defective sites of graphene, they likely contribute to Raman scattering both as on-site defects (caused by the binding of out-of-plane atoms to $\mathrm{sp}^{2}$-carbon) and hopping defects (produced by the displacement of carbon atoms). In the meantime, some $\mathrm{sp}^{3}$ cross-linked carbon atoms also likely contribute to $\mathrm{sp}^{3}$ defects. We note that after $10^{14} / \mathrm{cm}^{2} \mathrm{He}^{+}$ dose, the k-value of our 3D GA drops drastically to 4.41. This is surprising as one would expect the k-value shifts to higher values (i.e., closer to $\mathrm{k}=\sim 7$ ) $[11,26]$ as ion beam is known to continuously introduce vacancy-type defects into 3D graphene (see Fig. 1), which would have increased k-value. In addition to introducing point defects, here we suggest two plausible scenarios where ion-beam treatment may help to reduce the k-value. The first scenario may be due to the cleanup of functional groups on graphene sheets (for example, those bound on defective sites and long edges) such that it changes the nature of $\operatorname{sp}^{3}$-defects $(k=\sim 13)$, which could in turn lead to a drop of k-value. The second scenario could involve the easy knockout of $\mathrm{sp}^{3}$-type cross linking carbon atoms as they are intrinsically in high-energy state, leaving behind more stable $\mathrm{sp}^{2}$ carbon. This scenario may advertently help to reduce $\mathrm{sp}^{3}$-type defects and thus cause a lower k-value. The definitive mechanisms of above two scenarios remain to be investigated. After further treatment of 3D GA to higher doses of $10^{15} / \mathrm{cm}^{2}$ and $10^{16} / \mathrm{cm}^{2}$, interestingly, the k-value increases from 4.41 to 5.02, and finally to 5.26. This increasing trend seems to agree with the continuous increase of vacancy-type defects. Our studies thus suggest that ion-beam treatment on 3D GAs exhibit more complicated behavior than those seen in clean single graphene sheets. 


\subsection{Electrochemical behavior}

The electrochemical behavior of all four types of samples was investigated in the voltage range of 0.01-3.5 V at a current density of $50 \mathrm{~mA} / \mathrm{g}$. The lithiation/delithiation profiles of first and fifth cycles are shown in Fig. 5(a) and 5(b), respectively. For comparison, a reference voltage profile of graphite is also included. 3D GAs show drastically different charge/discharge behavior than that of graphite. Due to their large surface areas, the initial lithiation capacity of 3D GAs is observed to be as large as $\sim 3200 \mathrm{mAh} / \mathrm{g}$ for 1600C-GA sample, Fig. 5(a), which is nearly one order of magnitude higher than that of graphite $(\sim 372 \mathrm{mAh} / \mathrm{g})$. The reversible capacity of 1600CGA is $\sim 770 \mathrm{mAh} / \mathrm{g}$ [Fig. 5(b)] (twice the value of graphite), representing $\sim 23.8 \%$ of the lithiation capacity. A discharge plateau at $\sim 0.8 \mathrm{~V}$ is observed in all of our 3D graphene samples. Fig. 5(a) also indicates that the capacity of 3D GAs is mainly obtained below the discharge voltage of 1.0 $\mathrm{V}$, whereas in graphite little capacity is observed between $0.5-1.0 \mathrm{~V}$ discharge potential.

To understand the mechanistic processes of charge/discharge, we plot in Fig. 5(c) and 5(d) the differential capacity curves at different cycles (i.e., first and fifth cycle). During the first discharge (lithiation) of the GAs, Fig. 5(c), the voltage profile in the differential capacity curves shows several distinguishable reduction peaks at $\sim 1.1 \mathrm{~V}\left(\mathrm{R}_{1}\right), 0.8 \mathrm{~V}\left(\mathrm{R}_{2}\right)$ and $<0.5 \mathrm{~V}\left(\mathrm{R}_{3}\right)$ (note that $\mathrm{R}_{3}$ consists of several small peaks). The origin of higher-voltage peaks (i.e., $\mathrm{R}_{1}$ and $\mathrm{R}_{2}$ ) are currently not well understood; but might be related to the $\mathrm{Li}^{+}$binding with graphene defects/edges, the decomposition of electrolyte, and the formation of SEI [27, 28]. In the following fifth cycle, however, most reduction peaks gradually disappeared, Fig. 5(d), suggesting some nonreversible processes associated with these peaks [28]. Ion beam treatment apparently leads to reduced initial lithiation capacities, likely due to the surface area reduction or the cleaning of graphene surface area. The differential capacity of the delithiation for the first and fifth cycle shown in Fig. 5(e) and 5(f) indicates copious reaction peaks (each peak is labelled as an oxidation peak) for all graphene samples, suggesting multiple lithium binding sites in 3D graphene that are consistent with defective nature of all GAs (also see Table 1) and differs significantly from what is typically observed for graphite, which only shows a single reduction and oxidation peak, Fig. 5(c)-(f). We observe an upshift of the intercalation voltage at $\sim 0.2 \mathrm{~V}$ in ion beam treated samples, suggesting that ion bombardment might have modified the interlayer 
spacing of 3D GAs by removing functional groups. Earlier experiments have suggested that the enlarged interlayer spacing facilitates lithium intercalation into graphene layers [5].

The long-term cycling stability of all 3D GAs is shown in Fig. 6(a) and 6(b), including both lithiation and delithiation capacities. One signature of all GAs is the slow decay of both lithiation and delithiation capacities as the cycle number increases. This is not surprising as, without polymeric binders or other spacer materials, the slow restacking of graphene nanosheets in $3 \mathrm{D}$ space would occur, leading to a continuous reduction of total surface area. We therefore expect that these 3D GAs could act as excellent scaffold materials to fabricate composite electrodes such as metal oxides/graphene electrodes in which the restacking of graphene would become less severe due to the spacer roles of other materials [29]. Interestingly, the overall capacity of the as-synthesized 1600C-GA sample is measured to be higher than those after ion beam treatment. This observation appears to contradict the common belief that defects help lithium storage $[3,6]$. Previous experiments $[3,10]$ suggest a linkage between $I(D) / I(G)$ ratio of graphene and its lithium storage capacity, which does not hold true in our 3D GAs as ion-beam treated samples have higher $\mathrm{I}(\mathrm{D}) / \mathrm{I}(\mathrm{G})$ ratio but clearly lower capacity. Instead, we observe a new interesting phenomenon after ion beam treatment; that is, the Coulombic efficiency of all ion beam treated samples is substantially higher than that of the untreated sample, Fig. 6(b). In fact and as shown in Table 3, all ion-beam treated samples show high percentage reversible capacities compared to that of the untreated graphene. In addition, we observe that the He-15 sample exhibits higher reversible capacity than He-14 predominately due to the increment of vacancy density. This argues favorably that vacancy-type defects may help to enhance the reversible capacity in graphene, and that the absolute capacity difference before and after ion beam treatment is likely caused by the total surface area difference or the cleaning of the graphene surface. The 1600C-GA sample shows higher lithiation capacity likely attributed to the formation of more nonreversible SEI layers due to the higher surface area or more dirty surface, leading to artificially higher capacity but clearly lower Coulombic efficiency.

To investigate the capacity retention behavior during long-term cycling of 3D GAs before and after ion beam implantation, we normalize the lithiation capacity of each sample against its initial capacity value (i.e., percentage capacity retention), which is shown Fig. 7(a). Two rather interesting trends emerge. At low $\mathrm{He}^{+}$dose levels ( up to $\sim 10^{15} \mathrm{~cm}^{-2}$ ), we observe that two ion- 
beam treated samples (He-14 and He-15) exhibit better capacity retention than that of the untreated sample, demonstrating the beneficial roles of vacancy-type defects in helping improve capacity retention and Coulombic efficiency. This enhanced capacity retention ability in ionbeam treated samples may be attributed to more stable SEI layers. When ion beam dose reaches $\sim 10^{16} \mathrm{He}^{+} / \mathrm{cm}^{2}$, however, we observe a rapid capacity fading in ion-beam treated sample (He-16) despite its advantageously higher Coulombic efficiency. This behavior suggests that high dose ion beam may have deteriorated the strong cross-linked carbon-carbon bonds in 3D graphene, leading to the disintegration of 3D structure under electrochemical cycling. Nonetheless, these experiments demonstrate that ion-beam treatment can be applied to tune the electrochemical behavior of 3D GAs for LIBs.

Due to the ambiguity of $\mathrm{I}(\mathrm{D}) / \mathrm{I}(\mathrm{G})$ in depicting the defect structure in graphene [i.e., the same I(D)/I(G) value could correspond to two different defective stages, see Fig. 4(b)] and the fact that we observe a decreased total capacity with the increased $\mathrm{I}(\mathrm{D}) / \mathrm{I}(\mathrm{G})$ - a trend that is opposite to what has been reported in the literature [3, 10], we propose to apply $\mathrm{I}(\mathrm{D}) / \mathrm{I}\left(\mathrm{D}^{\prime}\right)$ ratio to qualitatively gauge the reversible capacity of 3D aerogels, as illustrated in Fig. 7(b). This is because this ratio is associated with the nature of defects. In general, we observe a better reversible capacity in all of our samples with the increased I(D)/I(D') values except for the H-16 sample due to the breakdown of 3D structures during the charge/discharge. This interesting trend argues favorably that defect-type is important to help maintain the reversible capacity of graphene materials. Since $\mathrm{sp}^{3}$-defects and vacancy-type defects have higher I(D)/I(D') ratios, they are likely to play more beneficial roles in enhancing the reversible capacity of graphene than boundary-type defects. In-depth theoretical understanding of above phenomenon is obviously needed in the future in order to fully decode the lithium storage mechanisms in defective graphene.

\section{Conclusion}

The defect structure and electrochemical behavior of 3D GAs are investigated before and after helium ion beam bombardment. In addition to introducing vacancy-type of defects, we find that ion beam treatment could also help to clean/alter functional groups on graphene surfaces and thus lead to complex defect evolution behavior under Raman spectroscopy. Vacancy-type defects are found to be beneficial in enhancing the Coulombic efficiency and reversible capacities of 
graphene. We identify a critical $\mathrm{He}^{+}$dose level of $10^{15} \mathrm{~cm}^{-2}$, at which a maximum capacity retention is observed when 3D GAs are applied as anodes for LIBs. A higher dose of $10^{16}$ $\mathrm{He}^{+} / \mathrm{cm}^{2}$ disintegrates the 3D graphene under electrochemical cycling, leading to a rapid capacity fading and a loss of long-term cycling stability. We find an increased percentage reversible capacity in 3D graphene with the increased $\mathrm{I}(\mathrm{D}) / \mathrm{I}\left(\mathrm{D}^{\prime}\right)$ ratios, suggesting that $\mathrm{sp}^{3}$-defects and vacancies are more effective for the reversible capacity of graphene than boundary-type defects. Our studies underscore the importance of defect types in influencing the lithium storage capacity of graphene materials, rather than their geometric location.

\section{Acknowledgement}

The work was performed under the auspices of the US Department of Energy by LLNL under contract No. DE-AC52-07NA27344. The project was supported by the Laboratory Directed Research and Development (LDRD) programs of LLNL (12-ERD-053, 15-ERD-022, and 13LW-099). The materials synthesis of this work was also supported by the UC Lab Fees Research Program under award 12-LR-235323. The authors wish to thank Drs. L.Y.L. Woo, A. Goldstein, and S. Shin for helpful discussion and TEM assistance. 


\section{References}

[1] Zhu Y, Murali S, Stoller MD, Ganesh KJ, Cai W, Ferreira PJ, et al. Carbon-Based Supercapacitors Produced by Activation of Graphene. Science 2011;332(6037):1537-41.

[2] Xu Y, Lin Z, Zhong X, Huang X, Weiss NO, Huang Y, et al. Holey graphene frameworks for highly efficient capacitive energy storage. Nat Commun 2014;5:4554.

[3] Pan D, Wang S, Zhao B, Wu M, Zhang H, Wang Y, et al. Li Storage Properties of Disordered Graphene Nanosheets. Chem Mater 2009;21(14):3136-42.

[4] Nardecchia S, Carriazo D, Ferrer ML, Gutierrez MC, del Monte F. Three dimensional macroporous architectures and aerogels built of carbon nanotubes and/or graphene: synthesis and applications. Chem Soc Rev 2013;42(2):794-830.

[5] Yoo E, Kim J, Hosono E, Zhou H-s, Kudo T, Honma I. Large reversible Li storage of graphene nanosheet families for use in rechargeable lithium ion batteries. Nano Lett 2008;8(8):2277-82.

[6] Liu Y, Artyukhov VI, Liu M, Harutyunyan AR, Yakobson BI. Feasibility of Lithium Storage on Graphene and Its Derivatives. J Phys Chem Lett 2013;4(10):1737-42.

[7] Liu Y, Wang YM, Yakobson BI, Wood BC. Assessing Carbon-Based Anodes for Lithium-Ion Batteries: A Universal Description of Charge-Transfer Binding. Phys Rev Lett 2014;113(2):028304.

[8] Goodenough JB, Kim Y. Challenges for Rechargeable Li Batteries. Chem Mater 2010;22(3):587603.

[9] Wang G, Shen X, Yao J, Park J. Graphene nanosheets for enhanced lithium storage in lithium ion batteries. Carbon 2009;47(8):2049-53.

[10] Fan ZJ, Yan J, Ning GQ, Wei T, Zhi LJ, Wei F. Porous graphene networks as high performance anode materials for lithium ion batteries. Carbon 2013;60:558-61.

[11] Eckmann A, Felten A, Mishchenko A, Britnell L, Krupke R, Novoselov KS, et al. Probing the Nature of Defects in Graphene by Raman Spectroscopy. Nano Lett 2012;12(8):3925-30.

[12] Zandiatashbar A, Lee GH, An SJ, Lee S, Mathew N, Terrones M, et al. Effect of defects on the intrinsic strength and stiffness of graphene. Nat Commun 2014;5.

[13] Mukherjee R, Thomas AV, Datta D, Singh E, Li J, Eksik O, et al. Defect-induced plating of lithium metal within porous graphene networks. Nat Commun 2014;5.

[14] Liu XH, Wang JW, Liu Y, Zheng H, Kushima A, Huang S, et al. In situ transmission electron microscopy of electrochemical lithiation, delithiation and deformation of individual graphene nanoribbons. Carbon 2012;50(10):3836-44.

[15] Zhang XT, Sui ZY, Xu B, Yue SF, Luo YJ, Zhan WC, et al. Mechanically strong and highly conductive graphene aerogel and its use as electrodes for electrochemical power sources. J Mater Chem 2011;21(18):6494-7.

[16] Chen Z, Ren W, Gao L, Liu B, Pei S, Cheng H-M. Three-dimensional flexible and conductive interconnected graphene networks grown by chemical vapour deposition. Nature Mater 2011;10(6):424-8.

[17] Charnvanichborikarn S, Shin SJ, Worsley MA, Kucheyev SO. Tailoring properties of carbonnanotube-based foams by ion bombardment. Appl Phys Lett 2012;101(10):103114.

[18] Charnvanichborikarn S, Worsley MA, Shin SJ, Kucheyev SO. Heavy-ion-induced modification of structural and mechanical properties of carbon-nanotube aerogels. Carbon 2013;57:310-6.

[19] Worsley MA, Kucheyev SO, Mason HE, Merrill MD, Mayer BP, Lewicki J, et al. Mechanically robust 3D graphene macroassembly with high surface area. Chem Commun 2012;48(67):8428-30.

[20] Worsley MA, Charnvanichborikarn S, Montalvo E, Shin SJ, Tylski ED, Lewicki JP, et al. Toward Macroscale, Isotropic Carbons with Graphene-Sheet-Like Electrical and Mechanical Properties. Adv Funct Mater 2014;24(27):4259-64. 
[21] Worsley MA, Pham TT, Yan A, Shin SJ, Lee JRI, Bagge-Hansen M, et al. Synthesis and characterization of highly crystalline graphene aerogels. Acs Nano 2014:DOI: 10.1021/nn505335u.

[22] Ziegler JF, Biersack JP, Littmark U. The stopping and range of ions in solids. New York: Pergamon; 1985.

[23] http://www.mtixtl.com/ (accessed 10/21/2014).

[24] Wang C, Li D, Too CO, Wallace GG. Electrochemical Properties of Graphene Paper Electrodes Used in Lithium Batteries. Chem Mater 2009;21(13):2604-6.

[25] Lucchese MM, Stavale F, Ferreira EHM, Vilani C, Moutinho MVO, Capaz RB, et al. Quantifying ion-induced defects and Raman relaxation length in graphene. Carbon 2010;48(5):1592-7.

[26] Cancado LG, Jorio A, Martins Ferreira EH, Stavale F, Achete CA, Capaz RB, et al. Quantifying Defects in Graphene via Raman Spectroscopy at Different Excitation Energies. Nano Lett 2011;11(8):3190-6.

[27] Lee E, Persson KA. Li Absorption and Intercalation in Single Layer Graphene and Few Layer Graphene by First Principles. Nano Lett 2012;12(9):4624-8.

[28] Pollak E, Geng BS, Jeon KJ, Lucas IT, Richardson TJ, Wang F, et al. The Interaction of Li+ with Single-Layer and Few-Layer Graphene. Nano Lett 2010;10(9):3386-8.

[29] Li N, Chen Z, Ren W, Li F, Cheng H-M. Flexible graphene-based lithium ion batteries with ultrafast charge and discharge rates. Proc Natl Acad Sci U S A 2012;109(43):17360-5. 
Table 1 Calculated binding energy $(\mathrm{eV})$ of lithium on graphene with various structural defects, referenced to bulk metallic lithium. Negative energies indicate stability with respect to lithium cluster formation. The data are taken from reference [6].

\begin{tabular}{ccllllllll}
\hline $\begin{array}{l}\text { Defect } \\
\text { type }\end{array}$ & Pentagon & Heptagon & $\begin{array}{l}\mathbf{5 - 7} \\
\text { Dislocation }\end{array}$ & $\begin{array}{l}\text { Stone- } \\
\text { Wales }\end{array}$ & $\begin{array}{l}\text { Mono- } \\
\text { vacancy }\end{array}$ & $\begin{array}{l}\text { 555-777 } \\
\text { Di- } \\
\text { vacancy }\end{array}$ & $\begin{array}{l}\text { Ad- } \\
\text { dimer }\end{array}$ & $\begin{array}{l}\text { Zig- } \\
\text { zag } \\
\text { edge }\end{array}$ & $\begin{array}{l}\text { Armchair } \\
\text { edge }\end{array}$ \\
\hline $\begin{array}{c}\text { Binding } \\
\text { energy } \\
\text { (eV) }\end{array}$ & -0.45 & +0.19 & -0.32 & 0.00 & -1.09 & -0.78 & -0.22 & -2.07 & -1.03 \\
\hline
\end{tabular}

Table 2 Physical properties of commercial electrolyte used in our experiments.

\begin{tabular}{cc}
\hline & Specifications \\
\hline Salt & LiPF6 \\
Organic solvent & EC: DMC: DEC (1:1:1 in volume) \\
Chromaticity & $\leq 50 \mathrm{Hazen}$ \\
Moisture & $\leq 20 \mathrm{ppm}$ \\
HF & $\leq 50 \mathrm{ppm}$ \\
Conductivity & $10.50 \pm 0.50 \mathrm{~ms} / \mathrm{cm} @ 25^{\circ} \mathrm{C}$ \\
Density & $1.23 \pm 0.01 \mathrm{~g} / \mathrm{ml}$ \\
\hline
\end{tabular}

Table 3 Reversible capacity of 3D graphene before and after ion-beam treatment. Data are presented as lithiation capacity $(\mathrm{mAh} / \mathrm{g}) /$ delithiation capacity $(\mathrm{mAh} / \mathrm{g}) /$ Coulombic efficiency.

\begin{tabular}{ccccc}
\hline Cycle No. & 1st & 4th & 10th & 20th \\
\hline 1600C-GA & $3230 / 770 / 23.8 \%$ & $783 / 592 / 75.6 \%$ & $513 / 434 / 84.6 \%$ & $408 / 355 / 87.1 \%$ \\
He-14 & $2211 / 613 / 27.7 \%$ & $532 / 446 / 84.0 \%$ & $382 / 347 / 90.9 \%$ & $304 / 288 / 94.9 \%$ \\
He-15 & $2253 / 630 / 28.0 \%$ & $550 / 457 / 83.0 \%$ & $399 / 364 / 91.3 \%$ & $334 / 309 / 92.5 \%$ \\
He-16 & $2542 / 648 / 25.5 \%$ & $483 / 403 / 83.5 \%$ & $313 / 285 / 91.1 \%$ & $235 / 218 / 92.8 \%$ \\
\hline
\end{tabular}




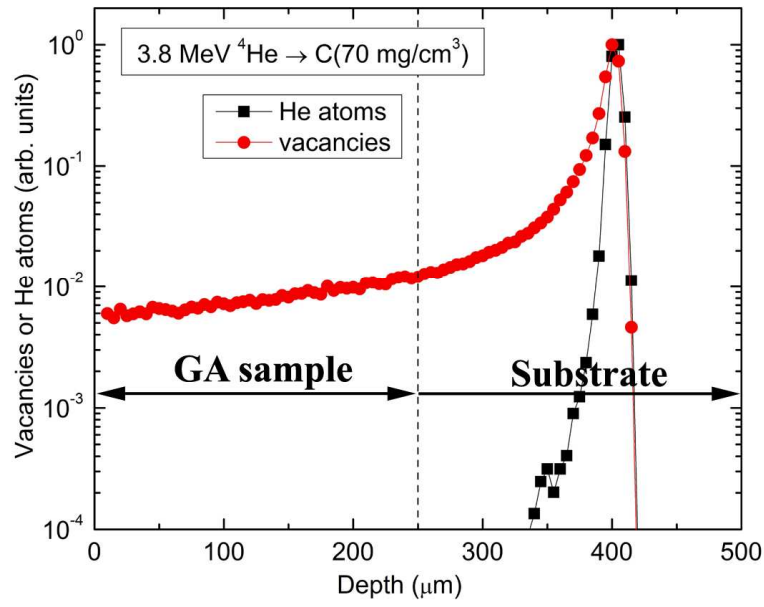

Figure 1 Calculated depth distributions of lattice vacancies and implanted He atoms for $3.8 \mathrm{MeV}$ $\mathrm{He}$ ion bombardment of 3D graphene with a density of $70 \mathrm{mg} / \mathrm{cc}$. The vertical dashed line indicates the thickness of the graphene bulk samples used in this study. 


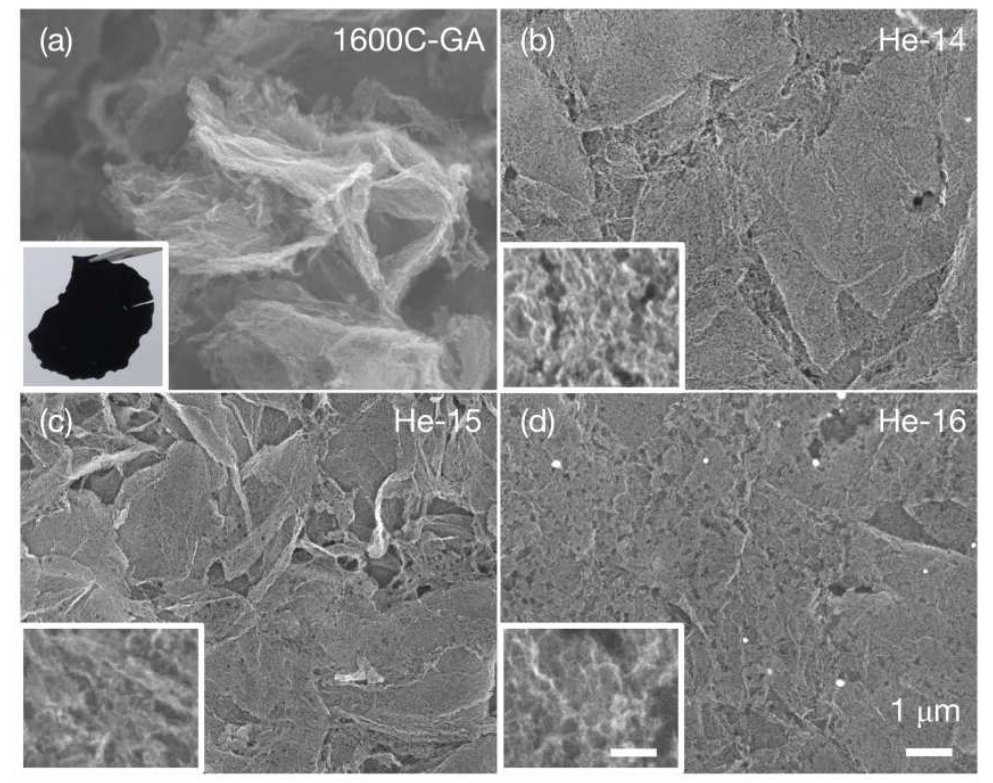

Figure 2 The morphologies of as-synthesized and He-ion irradiated graphene aerogels. (a) A top-surface SEM image of the pristine 1600C-GA. The inset is an optical image the sample disc with a thickness of $\sim 250 \mu \mathrm{m}$. (b)-(d) Top-surface SEM images of He-ion irradiated GA with the dose level of $\sim 10^{14} \mathrm{~cm}^{-2}, \sim 10^{15} \mathrm{~cm}^{-2}$, and $\sim 10^{16} \mathrm{~cm}^{-2}$, respectively. Inset images in (b)-(d) are zoomed-in images of respective samples with the same scale bar of $100 \mathrm{~nm}$. 

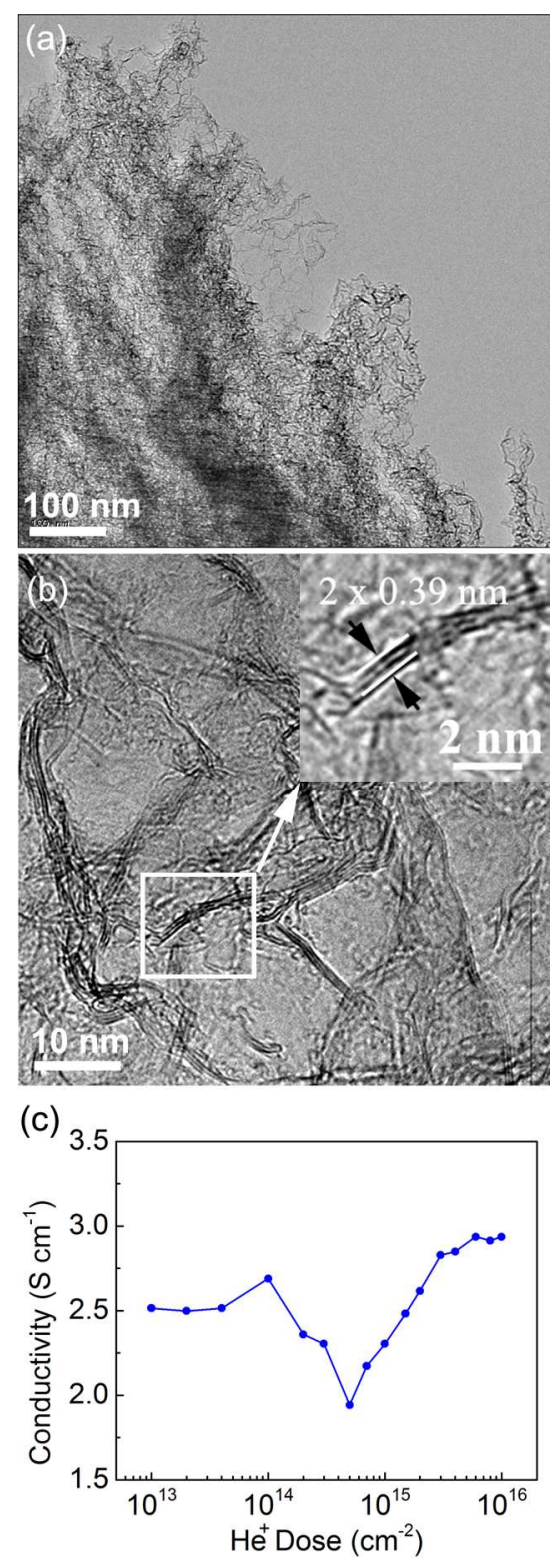

Figure 3 TEM micrographs of as-synthesized 3D graphene sample under (a) low- and (b) highmagnifications. The inset in (b) is a zoomed-in high-resolution TEM image showing the interlay spacing of graphene layers. (c) Electrical conductivity of 3D graphene aerogels as a function of $\mathrm{He}^{+}$dose. 


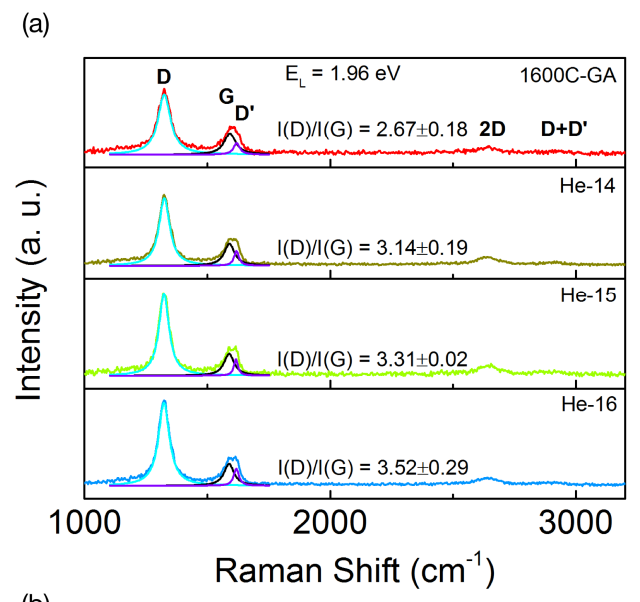

(b)

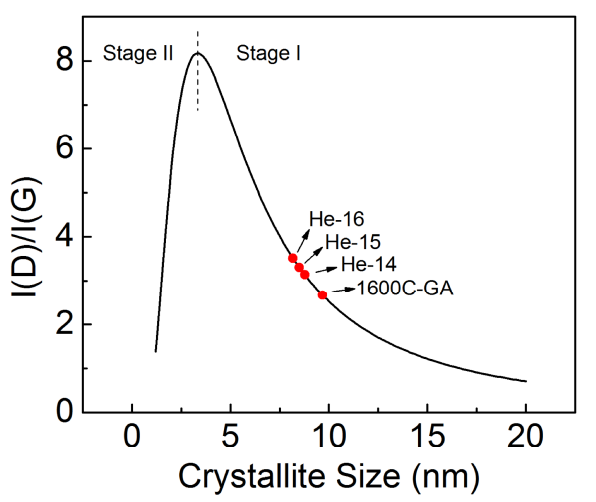

(c)

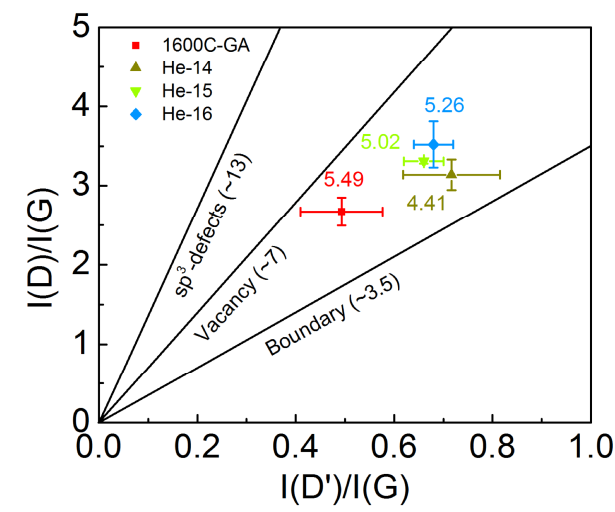

Figure 4 Raman analysis of the pristine and He-ion irradiated graphene aerogels. (a) Full Raman spectra show the fingerprint Raman shifts of graphene structure. The D, G, and D' peaks were fitted by Lorentzian functions. (b) Graphene domain size estimated from $\mathrm{I}(\mathrm{D}) / \mathrm{I}(\mathrm{G})$ ratios by using equation (2). The solid black line is the re-plot of the relation between $\mathrm{I}(\mathrm{D}) / \mathrm{I}(\mathrm{G})$ and domain size. (c) The plot of I(D)/I (G) ratio versus $I\left(D^{\prime}\right) / I(G)$. The slope $I(D) / I\left(D^{\prime}\right)$ defines the type of defects in graphene according to reference [11]. The $\mathrm{sp}^{3}$-defects, vacancy and boundary defects have slopes of $\sim 13, \sim 7$ and $\sim 3.5$, respectively. 
(a)

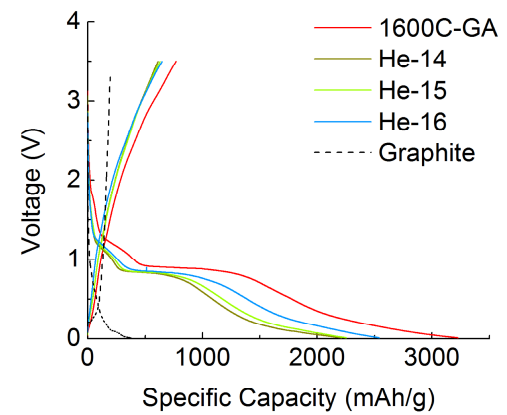

(c)

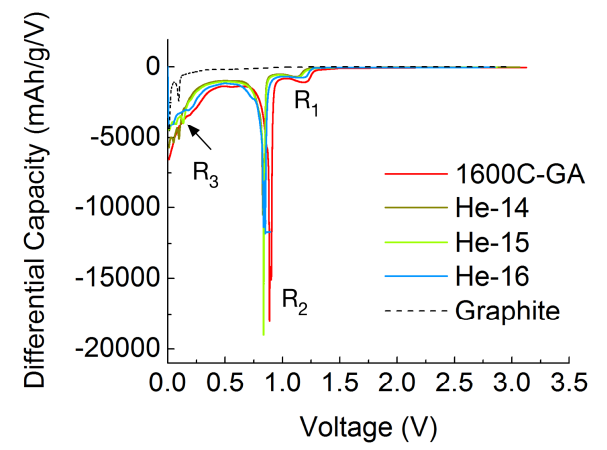

(e)

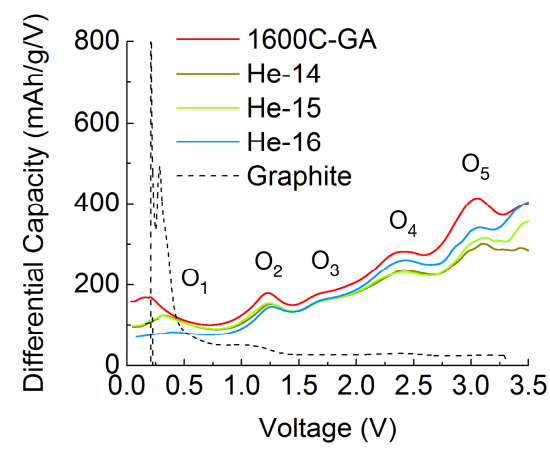

(b)

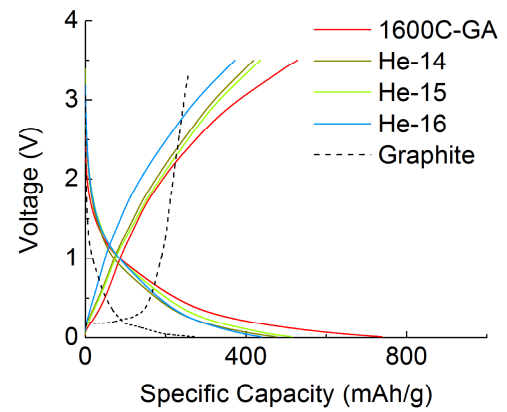

(d)



(f)

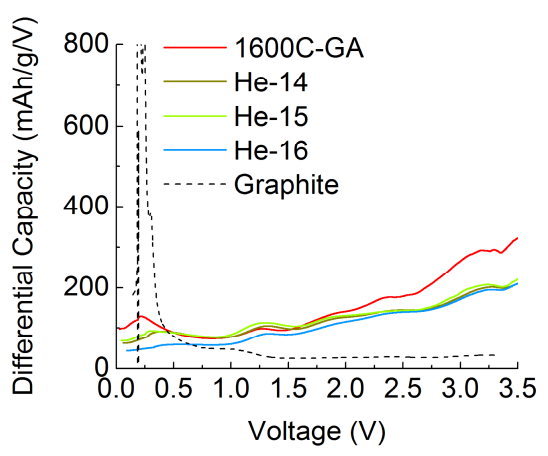

Figure 5 Charge/discharge voltage profiles and differential capacities for all graphene aerogels and reference graphite. Voltage profiles during (a) the first and (b) fifth cycles and the corresponding differential capacity curves during (c)-(d) lithiation and (e)-(f) delithiation processes of pristine and He-ion irradiated GAs, as well as graphite. 
(a)

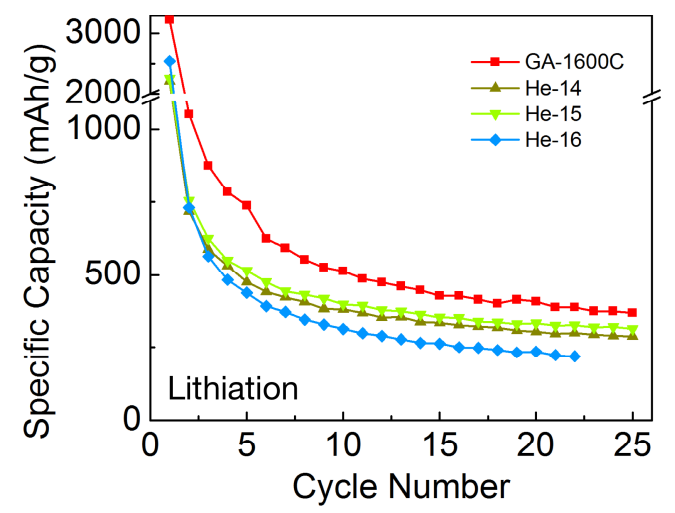

(b)

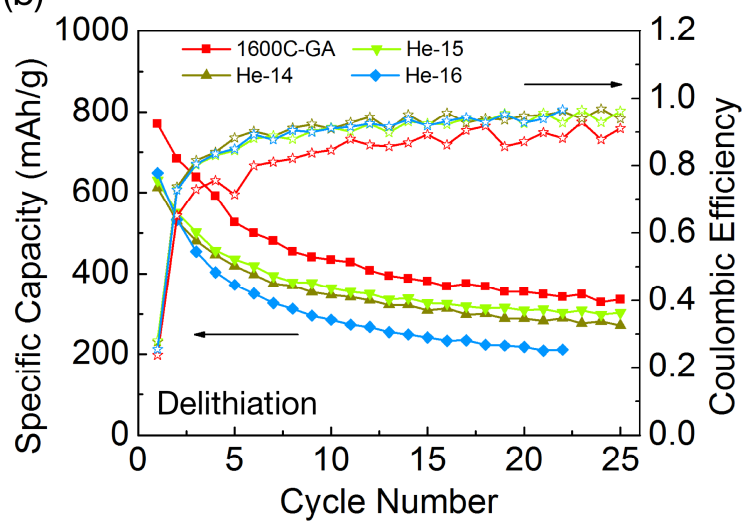

Figure 6 Long-term cycle stability and Coulombic efficiencies of various graphene aerogels. Specific capacities measured from (a) lithiation and (b) delithiation processes of pristine and Heion irradiated GAs at a current density of $50 \mathrm{~mA} / \mathrm{g}$. The corresponding Coulombic efficiencies are shown in (b). 
(a)

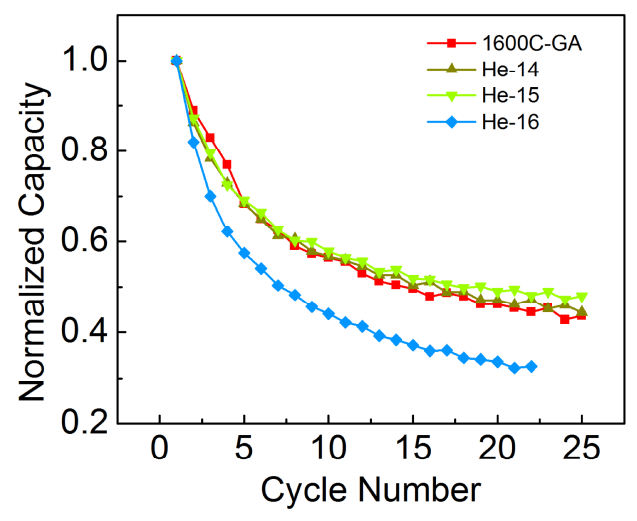

(b)

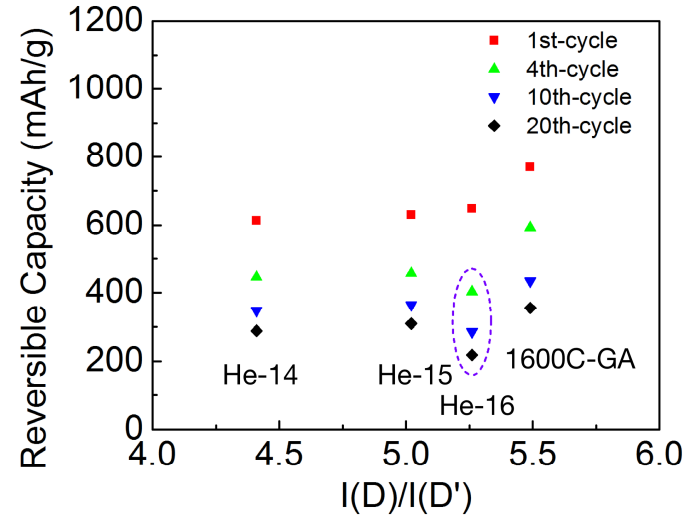

Figure 7 Capacity retention behavior in 3D graphene aerogels. (a) The normalized capacity calculated by dividing the delithiation capacity of each cycle to that of the first cycle. (b) The reversible capacity versus I(D)/I(D') ratio. The reversible capacity generally increases with the ratio of $\mathrm{I}(\mathrm{D}) / \mathrm{I}\left(\mathrm{D}^{\prime}\right)$. The data points inside the dashed oval are from the He-16 sample where the heavy ion dose appears to disintegrate the 3D structures. 